ISSN: 2277-3754

ISO 9001:2008 Certified

International Journal of Engineering and Innovative Technology (IJEIT)

Volume 10, Issue 4, October 2020

\title{
Global Video Stabilization with Truncated Motion Estimation Model and Motion Smoothening
}

Arti Makwana, Siddharth Singh Parihar

\begin{abstract}
Extensive use of low-cost handheld cameras triggers handshakes and camera gestures to the majority of captured images. Methods to eliminate undesired camera movements from Video sequences have been developed for Video Stabilization (VS). Movement of camera-related artifacts may also affect the efficiency of most $2 D$ video stabilization processes. By improving the motion estimation or motion fluidity process, the performance of the VS procedure can be improved. This article therefore proposes a global approach for video stabilization, which combines an efficient motion smoothing algorithm with a truncating motion calculation. Smoothing up of the high-frequency movements within frames achieves the stabilization. During the first step, the global movement estimate is selecting global motion vectors with the truncated Taylor series expansion. An FIR philter is used in this article to smooth global movement vectors by estimated motion vectors to stabilize shaky images. The proposed method eliminates undesirable movements efficiently and also conserves video information. Motion smoothening eliminates the loss of frame areas after stabilization. The method's efficiency is also compared with the latest global video stability results.
\end{abstract}

Index Terms - Video stabilization, Motion Smoothening, Taylor series, FIR Filter, IIR, Gaussian Kernel Filtering.

\section{INTRODUCTION}

The hand-held camera usually captures video from a standing or slow moving platform with small amount of rotations about the camera's optical axis. In the handheld case the camera is handled by the camera users (operator's) hands rather than to being mounted on a stand or any other base. Thus the captured videos suffer from inter frame unwanted motions. Therefore, video stabilization [1], [2] is becoming an indispensable technique for improving the quality of videos captured from these hand held cameras. The accuracy of the motion measurement must be as high as possible to achieve good VS. A broad range of methods are developed to evaluate motion [9 and 11]. The video may be processed as an images series or stream, called frames, in which each image is processed separately. However, using the Multi Frame processing, we can build successful algorithms using current temporal redundancies.

These algorithms [3, 4, 5, and 6], can broadly classify as; Motion compensated filtering and motion compensated predictions, motion segmentation and object tracking, video surveillance, video compression, video streaming and video stabilization.

Manuscript received: 22 September 2020

Manuscript received in revised form: 18 October 2020

Manuscript accepted: 02 November 2020

Manuscript Available online: 15 November 2020
The block diagram of video stabilization is presented in the Figure 1. There are three main stages of video stabilization process viz. motion estimation, smoothening, and compensation. Motion filtering is essential for the removal of the estimated motion vectors between the frames. During the smoothening jitters are also eliminated thus video is stabilized. In current past numerous low pass filters were designed as IIR, FIR, Kalman [1], [3], [5], and particle filter [7]. A method of the motion vector smoothening using first order IIR filters [1, 4] and Gaussian kernel smoothing [6] is used for filtering the estimated camera motions, and are defined as the weighted average value of the $\mathrm{k}$ previous and future frame motion vectors [6].

\section{CHALLENGES}

Video stabilization is a challenging task to implement. The major challenge is selection of the motion model because the simple motion models are inefficient for video with large scene depth, or when the object is closer to camera. Another major challenge is the extended period of exposure time or scene capturing time.

- The mechanical and optical image stabilization consumes large power thus battery life is reduced with these stabilization systems.

- The digital video stabilization (DVS) algorithms are computationally more complex than other stabilization methods.

- The video stabilization is most challenging task when multiple moving objects are there in the scene. Since objects are moving with different speed having different DOF, thus affected by varying blur. Thus efficient motion smoothening become difficult task in hand.

The basic stages of the video stabilization methods are shown in the Figure 1. It is generally a four stage approach

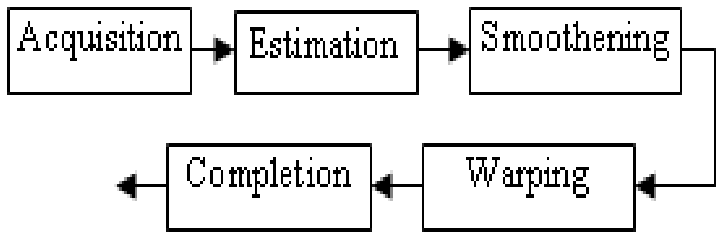

Fig.1.Blocks of the standard Video stabilization methods.

\section{A. Contribution of paper}

In this paper an approach of $\mathrm{n}^{\text {th }}$ order FIR filter design and analysis is presented to smooth the motion vectors estimated globally. A differential global motion vector estimation method based on hierarchical truncated Taylor 
ISSN: 2277-3754

\section{ISO 9001:2008 Certified \\ International Journal of Engineering and Innovative Technology (IJEIT) \\ Volume 10, Issue 4, October 2020}

series expansion [3] is used to determine motion vectors. The performance is compared with the IIR filter as in [3 and 1]. The paper is focused to remove the inter frame motions for videos with relatively shaky motions. The proposed method smoothen the motion vectors using adaptive coefficients of the FIR filter. Thus at the end it results less missing frame areas in the accumulative motion case. This in turn reduces computation coast.

\section{LITERATURE REVIEW}

Lot of video stabilization (VS) methods has been designed in last two decades. The VS techniques are classified to $2 \mathrm{D}$ or $3 \mathrm{D}$ stabilization. The $2 \mathrm{D}$ stabilization methods are relatively less complex and efficient to estimate and smooth camera motions. Global motion estimation methods are popular in recent studied but are complex. Most of 2D VS methods assume the 2D affine model due to its low complexity.

Lei Zhang et al [1] have recently designed a global VS method using quadratic energy minimization problem. They have used the global similarity invariant motion model. This work has motivated for working towards the global method of VS, Liu Wen et al [4] have presented the Feature based VS method for the ship board video. They uses adaptive Harris algorithm for motion estimation and then Fourier series based curve fitting is used for removing the unwanted camera motions. But method was feature based and efficiency may vary with the different kind of edges in video objects. Hany Farid [7] had first proposed the basic truncated Taylor series expansion method for global motion estimation by minimizing the inter frame quadratic error equation. They proposed temporal derivatives using 1D separable kernel filters for motion estimation.

Yang et.al [10] in 2006 proposed the adaptive image stabilization algorithm using multi-resolution sub-sampled based block motion estimation to determine global motion vectors. They designed adaptive IIR filter for smoothening the inter frame jitters. An author also uses a 2D compensation method to eliminate motion orthogonal to the panning direction. A. J. Crawford et al [11] have initially proposed a simplest method to predict smooth camera path using a low-pass IIR filter. A. Litvin et al [12] have proposed a probabilistic based motion smoothening method for video stabilization using the adaptive Kalman filter application. Kalman filter was used for estimating the smooth camera trajectory. Richard et al [14] have designed an FIR filter for the image stabilization but have demonstrated large missing image areas. The performance is compared with the Kalman filter for stabilization. Feng Liu et al [15] propose a 3D content preserving features based global motion estimation method and have also made input videos global with content preserving videos 2 and 4 . In this paper an optimum FIR filter is proposed to smooth the estimated global motion vectors. The coefficient and order of filter are varied for smoothening.

\section{PROPOSED METHODOLOGY}

Block diagram of proposed VS algorithm is presented in Figure 2. In proposed method global motion is estimated based on the hierarchical motion estimation. Then for motion smoothening FIR filter is proposed to use. The proposed motion smoothening method significantly removes the unwanted inter frame motions.

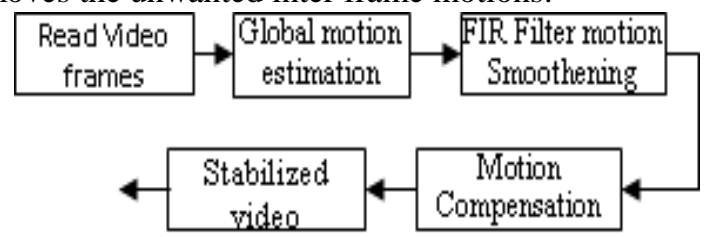

Fig.2.Proposed block diagram..

\section{A. Truncated Global Motion Estimation}

In this paper a truncated Taylor series based global motion estimation is proposed to determine motion between inter frames of 2D videos. The 2D motion is modeled using the affine motion model for inter frames defined as $f\left(x_{x}, y, t\right)$ and $f\left(x_{v} y_{s} t-1\right)$. Global motion vectors can be estimated using truncated Taylor series expansion as in [7]. The motion between frames are formulated as

$$
\begin{aligned}
& f(x, y, t)=f\left(m_{1} x+m_{2} y+m_{z}, m_{2} x+m_{4} y+m_{1}, t-1\right) \\
& \quad A=\left(\begin{array}{cc}
m 1 & m 2 \\
m a & m
\end{array}\right) \text { and } \bar{T}=\left(\begin{array}{c}
m 5 \\
m 6
\end{array}\right)
\end{aligned}
$$$$
\text { Where }
$$

In order to estimate the affine parameters, we define the following quadratic error function to be minimized.

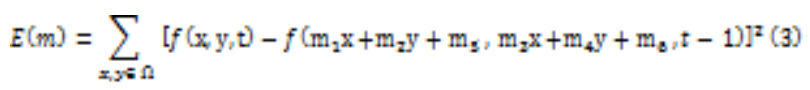

This can be approximated as first order truncated Taylor expansion (Ref $[3,7]$ for detail) as

$$
E(m)=\sum_{x=\Omega}\left[k-C^{T} m\right]^{2}
$$

This equation is solved for motion vector estimation and the estimated global vectors in the $\mathrm{x}$ and $\mathrm{y}$ direction are given

$$
G M V(t)=\left[G M V_{X}(t)_{e} G M V_{Y}(t)\right]
$$

\section{B. FIR Motion Smoothing}

Where the accumulated smoothened motion vectors $\operatorname{ASMV}(t)$ are defined a s;

$$
\operatorname{ASMV}(t)=\left\{A S M V^{x}(t), A S M V^{2}(t)\right\}
$$

In order to implement a FIR filter the filter's impulse response is convolved with the original input signal. These filters are used by many researchers [9, and 14] in past to smooth motion in image/video sequences. Filter utilizes the information available in finite number of recent and future global motion vectors. FIR filters smoothens the data vector $x$ with the filter described by numerator and denominator coefficient vectors ${ }^{a}$ and ${ }^{b}$ respectively Filter depends on the current and past four frames motion vectors. Frames are filtered with filter coefficients 
ISSN: 2277-3754

ISO 9001:2008 Certified

International Journal of Engineering and Innovative Technology (IJEIT)

Volume 10, Issue 4, October 2020

$b 0=2^{-1}{ }_{x} b 1=2^{-2}{ }_{x} b 2=2^{-a}{ }_{x} b 3=2^{-4}{ }_{x}$ and $b 4==2^{-4}$. (7 )

The number of past frames can be modified as needed and the filter coefficients may be adjusted accordingly. Filtering is performed as;

$$
B M V_{f a t e r}\left(n_{i} b l k\right)=\sum_{K=0}^{a} b_{k} * B M V_{\text {sld }}\left(n-k_{2} b I k\right)
$$

An FIR filter requires more computation time and memory than IIR filter but is efficient. In this paper for the performance comparison FIR filter with numerator coefficient vectors $a$ is set to 1 to normalize the filter coefficients, and denominator coefficient $b$ is set to 0.2 and 2 with standard filter order of 31 .

\section{COMPENSATION OF MOTION}

In this paper we choose the motion compensation from Gaussian kernel filtering us. Using Gaussian filtering eliminates areas that are incomplete. The offset vectors are calculated from frame to frame,

$$
C_{t}=\sum_{i \in \mathbb{N}_{\mathrm{r}}} T_{j}^{i} * G(k)
$$

Where, $G(k)$ is kernel filter as defined by [3], and $N_{t}$ is the neighborhood given as;

$$
N_{t}=m: k-n \leq m \leq k+n
$$

The motion compensated frames $f_{t}^{c}$ can be warped from the original frame $f_{t}$ by

$$
f_{t}^{c}\left(x, y_{s} t\right)=C_{t} * f_{t}\left(x_{s} y_{s} t\right)
$$

Thus using the $\mathrm{Ct}$ coefficients compensated frames are obtained.

\section{RESULTS AND DISCUSSION}

The $2.4 \mathrm{GHz}$ and MATLAB Core2 Duo processor is used to develop the proposed global system of video stabilization. A motion smoothing method for extracting movement vectors by filtering global motion vectors is being proposed. The proposed method is contrasted to current methods, as seen in [1] and[3]. The method is used in this regard. As seen in the Feng Lu[15], open input videos are being checked for the efficiency of the proposed video stabilization algorithm. The two video sequences input are 3D video content 2 and 3D video 4 content, as shown in Figure 3.
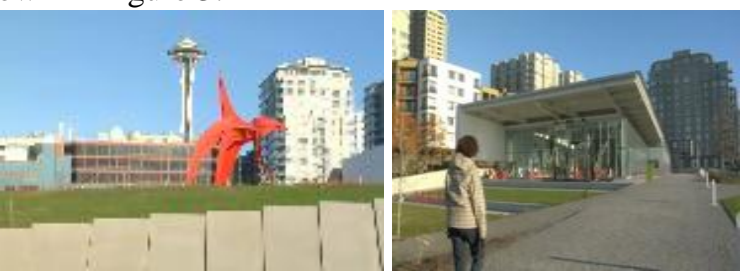

Fig 3. Input videos a) Content preserving video 2, b) Content preserving video 4 [15]

Videos are with slow and largest shaky motion of object and camera moving with speed around $<10 \mathrm{~m} / \mathrm{s}$

\section{Motion Smoothening}

The proposed method measures, using a differential motion assessment, the accumulated world movement vectors in $\mathrm{X}$ and $\mathrm{Y}$ directions. Then FIR coefficients are chosen in accordance with eq 8) to smooth the approximate vectors of motion $\mathrm{X}$ and $\mathrm{Y}$. Filter smoothes inter frame movements and thus stabilizes the video effectively. The FIR philter proposed is equipped with 31 st order adaptive FIR philtres. The vector of the filtered numbering coefficient $=0,2$ is normalized to $\mathrm{a}=1$ as in Figure 4 with the Denominator Coefficient. Compared with the traditional Deshaker method [16], the results of the proposed approach are as shown in Figure. 4.

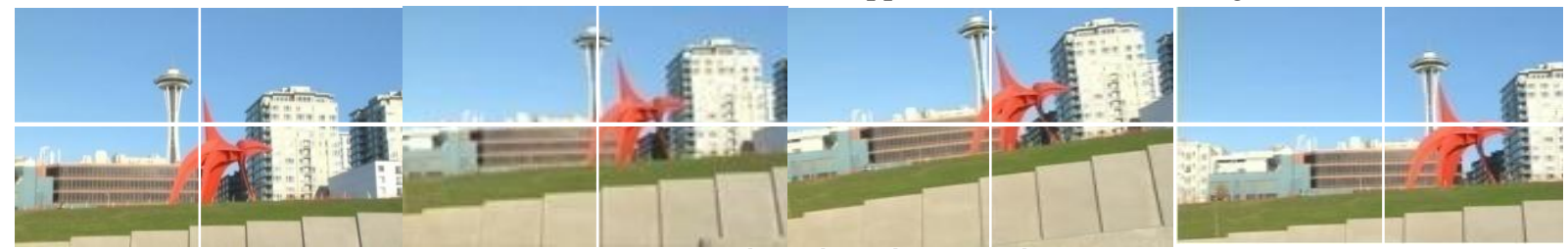

a) Input video frames $\left(25^{\text {th }}, 50^{\text {th }}, 75^{\text {th }}\right.$ and $\left.100^{\text {th }}\right)$ Feng Liu [15]

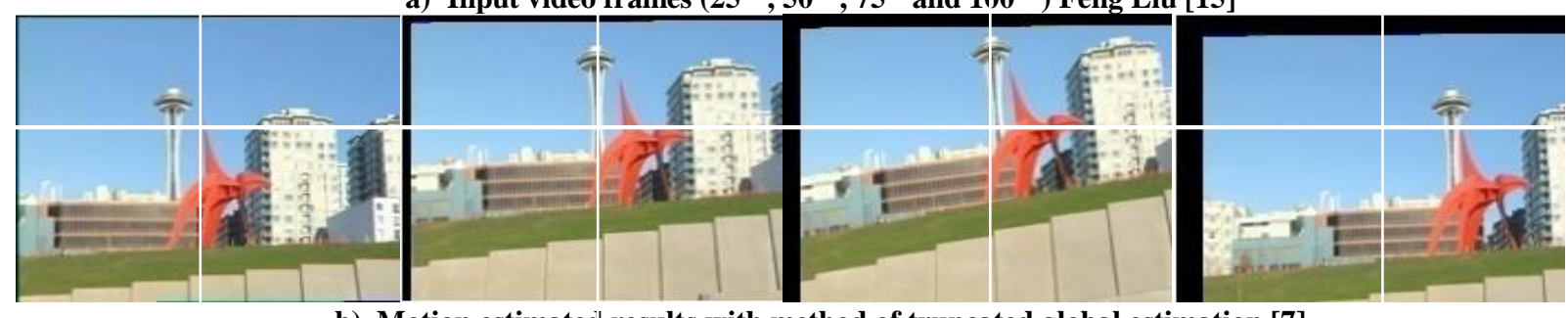

b) Motion estimated results with method of truncated global estimation [7]

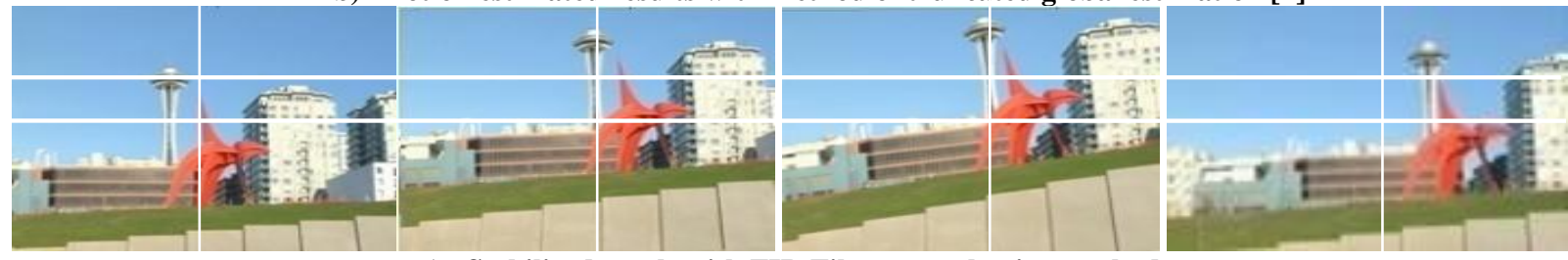

c) Stabilized result with FIR Filter smoothening method 


\section{DIEIT}

ISSN: 2277-3754

\section{ISO 9001:2008 Certified}

International Journal of Engineering and Innovative Technology (IJEIT)

Volume 10, Issue 4, October 2020

Fig.4 Results of the stabilized results with proposed FIR filter method (for Content 3D video 2 )
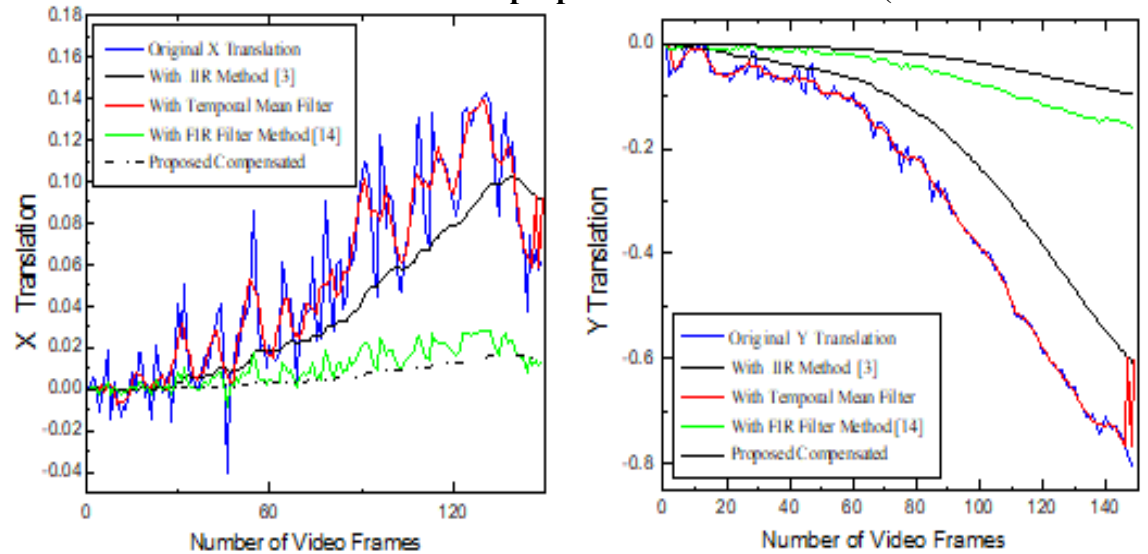

Fig. 5. Comparison of smoothen $X$ and $Y$ translation with various motion smoothening method for Content 3D video 4

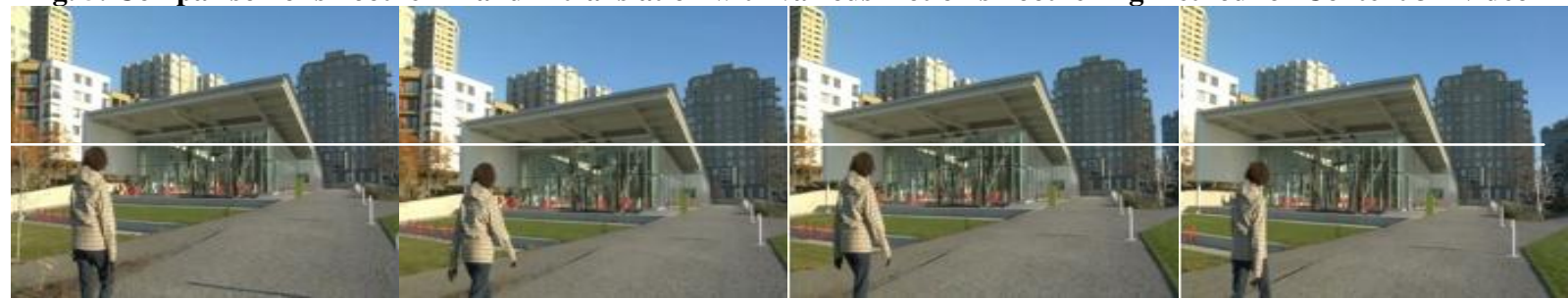

a) Input video frames $\left(25^{\text {th }}, 50^{\text {th }}, 75^{\text {th }}\right.$ and $\left.100^{\text {th }}\right)$ Feng Liu [15]

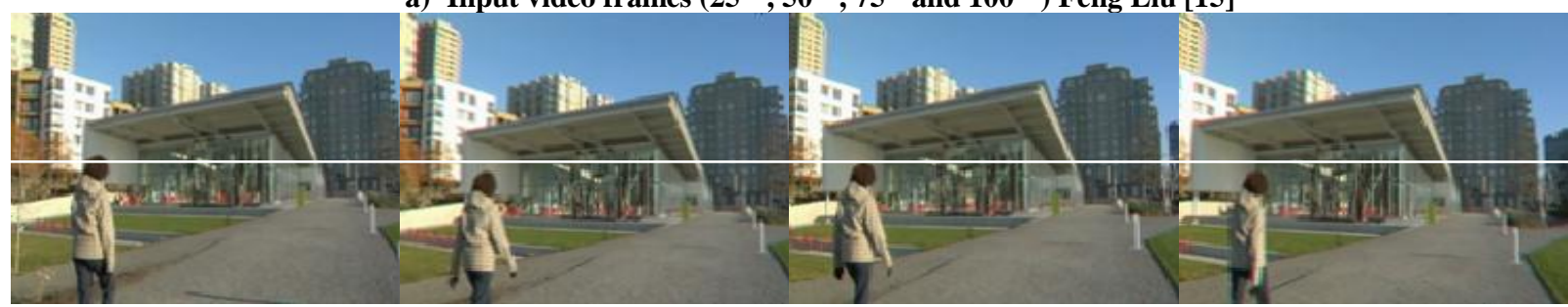

b) Stabilized result with temporal mean filtering method

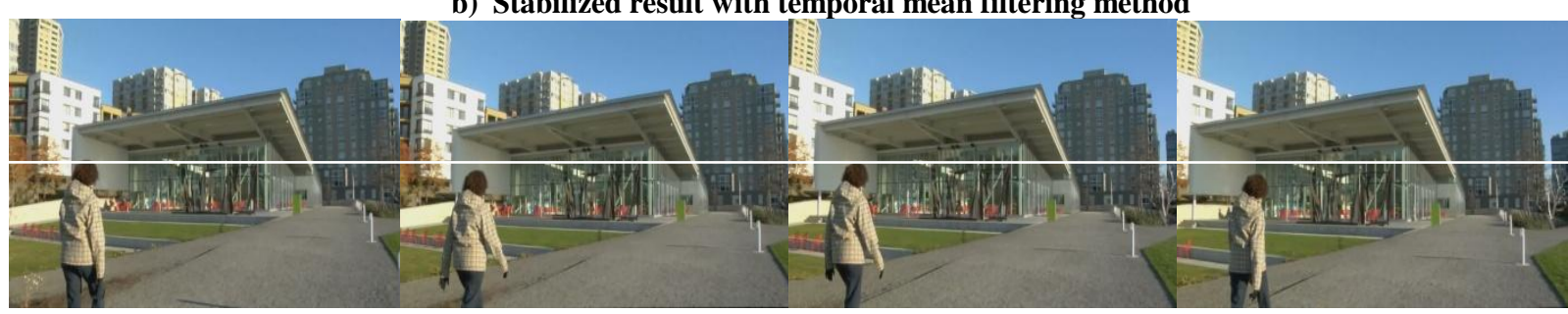

c) Stabilized with Deshaker method [16]

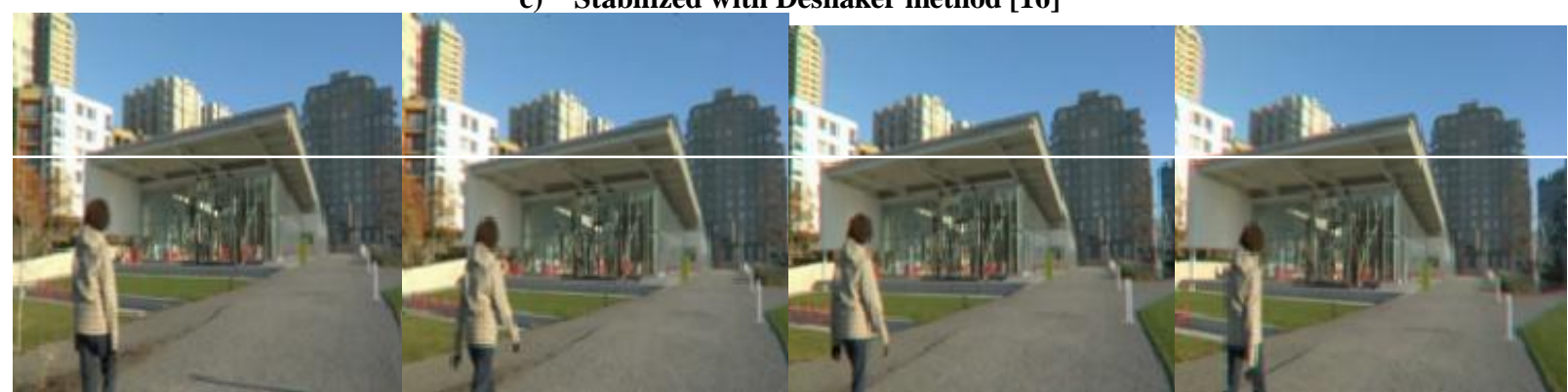

d) Stabilized result with proposed IIR smoothening method [3]

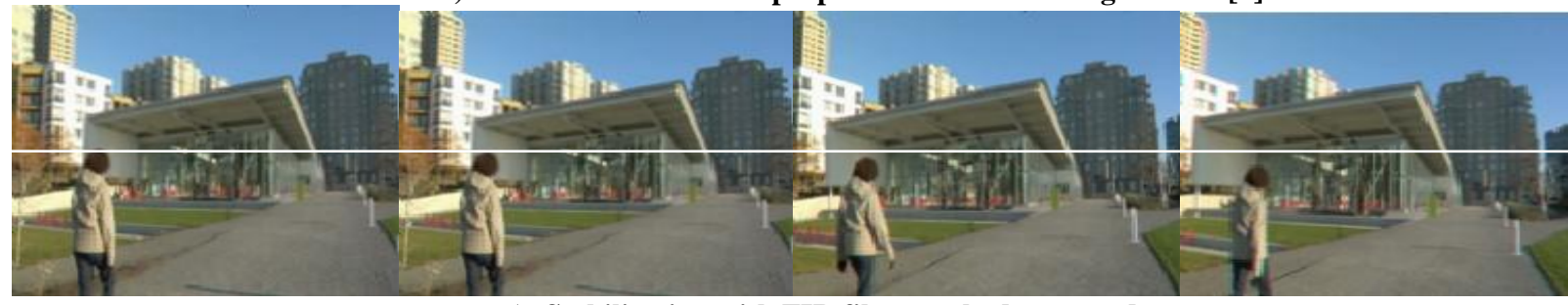

e) Stabilization with FIR filter method proposed

Fig 6.Comparison of results of various stabilize motion smoothening frames for Content 3D video 4 Feng Liu [15] 
ISSN: 2277-3754

ISO 9001:2008 Certified

International Journal of Engineering and Innovative Technology (IJEIT)

Volume 10, Issue 4, October 2020
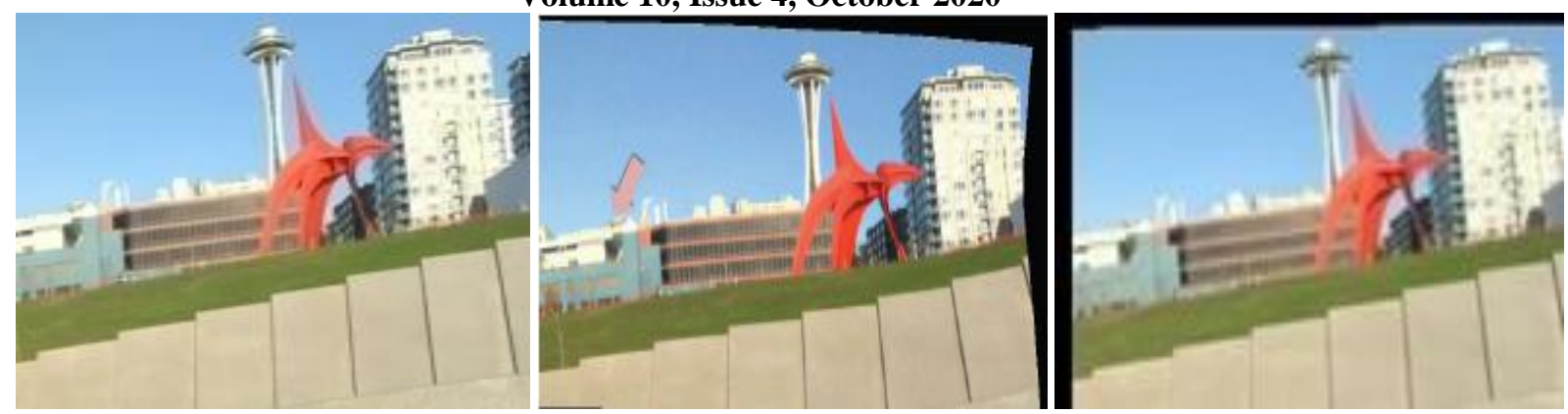

Fig. 7. Comparison of Global vector stabilization a) $75^{\text {th }}$ Frame b) Result of Lei Zhang, et al [1] c) result of estimation with proposed truncated frame error method

The results comparison of smoothen vectors as $\mathrm{X}$ and $\mathrm{Y}$ translation with different motion smoothening method for Content 3D video 4 video is presented in the Figure 5 below. In this video a person is walking front towards the building. The respective $25^{\text {th }}$ frames with mean, IIR, Desheker and FIR filter are shown in the Figure 6 for stabilizing Content 3D video 4 as referred to Feng Lu [15].

In the Figure 6 comparing the frames with respect to the white line in the center of frame it can be observed the proposed FIR filtered frames are better stabilized. As it gives complete frame areas and compared to Deshaker where frame is cropped on top. The brightness of the FIR filter is much better then comparative to IIR filtered method.

\section{CONCLUSIONS AND FUTURE WORK}

In this paper truncated inter frame motion estimation along with FIR filter to smoothen the motion is proposed. The differential global motion vectors are accumulated and filtered fro stabilizing the video. In order to compensate the missing frame areas the Gaussian compensation is used. The performance of the proposed method is compared with the state of art filters with respect to proposed global motion estimation method. Experiments have been conducted on a wide variety of video sequences. Performance is compared based on smote=then $\mathrm{X}$ and $\mathrm{Y}$ translation vectors using IIR, Deshaker and FIR filer and temporal mean filters.

The proposed method of motion smoothening with the FIR filter performs better for various types of motion and jitters. It can be seen that visual quality of frames are also relatively better in FIR filtered results. Although compared to IIR filter the FIR filter is slightly computationally costly.

\section{REFERENCES}

[1] Lei Zhang, Qian-Kun Xu, and Hua huang, "A Global Approach For Fast Video Stabilization", IEEE Transactions On Circuits System And Video Technology, Vol. 27, No. 2,pp:225-235,Feb 2017.

[2] Pyung Soo Kim, "FIR filtering based image stabilization mechanism for mobile video appliances" Proceeding CIS'04 Proceedings of the First international conference on
Computational and Information Science pp:1106-1113, 2004.

[3] P. Rawat, Jyotu Singhai, "Adaptive Motion Smoothening for Video Stabilization," International Journal of Computer Applications (0975 - 8887) Volume 72- No.20, pp:14-20, June 2013.

[4] Liu Wen, Zhang Yingjun, Yang Xuefeng, "A Feature -based Method for Shipboard Video Stabilization" IEEE 2nd International Conference on Electronic Information and Communication Technology, China, pp: 315-322, 2019.

[5] Zainab J. Ahmed, loay E.Goerge, bushra A. Sultan, "New Predictive Block Matching Searching Algorithms and Hybrid Predictive Search System", International Journal of Engineering and Innovative Technology (IJEIT) Volume 4, Issue 1, pp:230-235, July 2014.

[6] Hiroko Komai, Takayoshi Tezuka, Takeshi Hashimoto, András Rövid, Kaeko Yamashita, "Stereo Vision Based Therapeutic Massage Evaluation System", International Journal of Engineering and Innovative Technology (IJEIT) Volume 4, Issue 7, pp:25-30, January 2015.

[7] Hany Farid and J. B. Woodward, "Video stabilization and Enhancement", TR 2007-605, Dartmouth College, Computer Science, pp: 1-12, 1997.

[8] Pyung Soo Kim, "FIR filtering based image stabilization mechanism for mobile video appliances", Computational and Information Science Lecture Notes in Computer Science Volume 3314, pp 1106-1113, 2005.

[9] Jyoti Singhai, Paresh Rawat, "Review of motion estimation and video Stabilization technique For hand held mobile video" International Journal of Signal \& Image Processing (SIPIJ), Vol.2, No.2, pp:159-168, June 2011.

[10] Shih-Hsuan Yang, Fu-Min Jheng, "An Adaptive Image Stabilization Technique", IEEE International Conference on Systems, Man and Cybernetics, SMC '06. Volume: 3, pp: 1968-1973, 2006.

[11] A. J. Crawford, H. Denman, F. Kelly, F. Pitie, A. C Kokaram, "Gradient based dominant motion estimation with integral projections for real time video stabilization" IEEE International Conference on Image Processing. New Jersey, pp. 3371-3374, 2004.

[12] Martin Kumm, Diana Fanghanel, Konrad Moller, Peter Zipf1 and Uwe Meyer-Base, "FIR filter optimization for video processing on FPGAs", EURASIP Journal on Advances in Signal Processing 2013:111, pp:1-18, 2013.

[14] Richard Y. Park, Jung Min Pak, Choon Ki Ahn, and Myo Taeg Lim, "Image Stabilization Using FIR Filters", 15th 
ISSN: 2277-3754

ISO 9001:2008 Certified

International Journal of Engineering and Innovative Technology (IJEIT)

Volume 6, Issue 8, February 2017

International Conference on Control, Automation and

Systems (ICCAS 2015) Oct. 13-16, pp:1234-1237, 2015.

[15] Liu, F., Gleicher, M., Jin, H., \& Agarwala, A. "Content-Preserving Warps for 3D Video Stabilization" ACM Transactions on Graphics, Vol. 28, No. 3, pp:1-10, August 2009.

[16] J. Dong and H. Liu, "Video Stabilization for Strict Real-Time Applications," in IEEE Transactions on Circuits and Systems for Video Technology, vol. 27, no. 4, pp. 716-724, April 2017.

[17] Q. Xie, X. Chen, L. Zhang, A. Jiang and F. Cui, "A Robust and Efficient Video Anti-Shaking Algorithm for Low-End Smartphone Platforms," in IEEE Transactions on Consumer Electronics, vol. 65, no. 1, pp. 1-10, Feb. 2019.

[18] A. Yousaf, M. S. Hanif, M. J. Khan, M. Iqbal and K. Khurshid, "Robust and computationally efficient online image stabilization framework based on adaptive dual motion vector integration," in IET Computer Vision, vol. 13, no. 5, pp. 461-468, 2019. 